\title{
Seismo-Microplasticity phenomenon in the rocks ${ }^{*}$
}

\author{
Eduard Innokentevich Mashinskii
}

Institute of Petroleum Geology and Geophysics, Siberian Branch of the RAS, Novosibirsk, Russia; MashinskiiEI@ipgg.nsc.ru

Received 26 November 2009; revised 8 January 2010; accepted 30 January 2010.

\begin{abstract}
The seismic records of borehole-to-borehole measurements on frequency of $200 \mathrm{~Hz}$ in the microstrain range have been analysed. Microplasticity manifestations caused by seismic wave are detected on seismic records. It is the ladder-like stepwise change in amplitude course in some parts of the seismic trace. The step duration (time plateau) presents the amplitudedependent time delay that shifts the arrival time and protracts pulse front. The microplastic process occurs owing to the anomalous realignment of the internal stresses on the microstructural defects in "elastic" domain. Result is the useful contribution for improvement of the theory of wave attenuation in the rocks. It can also be used in solving the applied problems in material science, seismic prospecting, diagnostics etc.
\end{abstract}

Keywords: Anelasticity; Deformation And Time Delay; Anelastic Seismic Parameters; Amplitude Dependence Of Wave Velocity And Attenuation

\section{INTRODUCTION}

Perfection of the wave attenuation mechanisms is up to now one of main tasks in Earth's sciences. For understanding attenuation mechanisms, the new knowledge is necessary about the rock anelasticity. Viscoelastic model of standard linear solid well describes the dispersion and relaxation but insufficiently correctly explains, for example, the amplitude-dependent effects. So, there are some contradictions relative to character of amplitude dependences of wave velocity and attenuation. There are data about decrease of wave velocity and increase in attenuation with increasing amplitude [1-4]. However, there are also facts when increase in the strain amplitude leads on the contrary to the increase in wave velocity and decrease in attenuation [5-9].

Detection of the quasi-static rock microplasticity has

*This work was performed with the support of the Russian Fund of Fundamental Researches, grant N 05-09-00405. strengthened supposition about some unknown factor in charge of the amplitude dependence of seismic parameters [10-12]. It is necessary to mark that in Earth's sciences the microplastic anelasticity is not yet the generally accepted fact as against solid-state physics. In contrast to the viscoelasticity, microplasticity appears only when stress reaches critical value. The microplastic strain can increase and decrease, appear and vanish during increasing stress. The amplitude-dependent effects in rocks and quartz crystals were interpreted as indirect attributes of microplasticity that is possible even on the small strain amplitudes $[13,14]$. Assumption about microplasticity processes during seismic wave propagation was also made in works [15-16].

The direct testimony of seismic microplasticity was received in borehole-to-borehole measurements during the amplitude effect study [17]. This paper describes microplasticity manifestations detected on the seismic traces in result of the detailed analysis of the field materials.

\section{EXPERIMENTS AND DATA ANALYSIS}

The propagation of seismic pulse with diverse amplitudes in the area between two boreholes was studied. The experiments were performed in the Bystrovka research area. The measuring instruments were mounted in two boreholes $110 \mathrm{~mm}$ in diameter and $12 \mathrm{~m}$ depth spaced $7 \mathrm{~m}$. A source was located in one of the boreholes and a receiver, in the other. The source in borehole 1 and receiver in borehole 2 were successively installed at depth of 2, 6 , and $10 \mathrm{~m}$. Detailed description is in the work [17]. The upper part of the section is comparatively homogeneous and is composed of loams as far as a depth of a few tens of meters. Rock is partially water-saturated as far as $8.5 \mathrm{~m}$ with compressional wave velocity $\mathrm{Vp}=240-300 \mathrm{~m} / \mathrm{sec}$. After the depth $-9 \mathrm{~m}$ the wave travels in completely water-saturated loams with $\mathrm{Vp}=1500 \mathrm{~m} / \mathrm{sec}$.

The measurements were made in accordance with the following procedure. The source and the receiver were successively located at depths of 2,6 , and $10 \mathrm{~m}$ in the diverse combinations. Such source-receiver configuration enables to study the pulse propagation in the different direction. Basic seismic records were made on the location of source-receiver in the lateral (horizontal) 


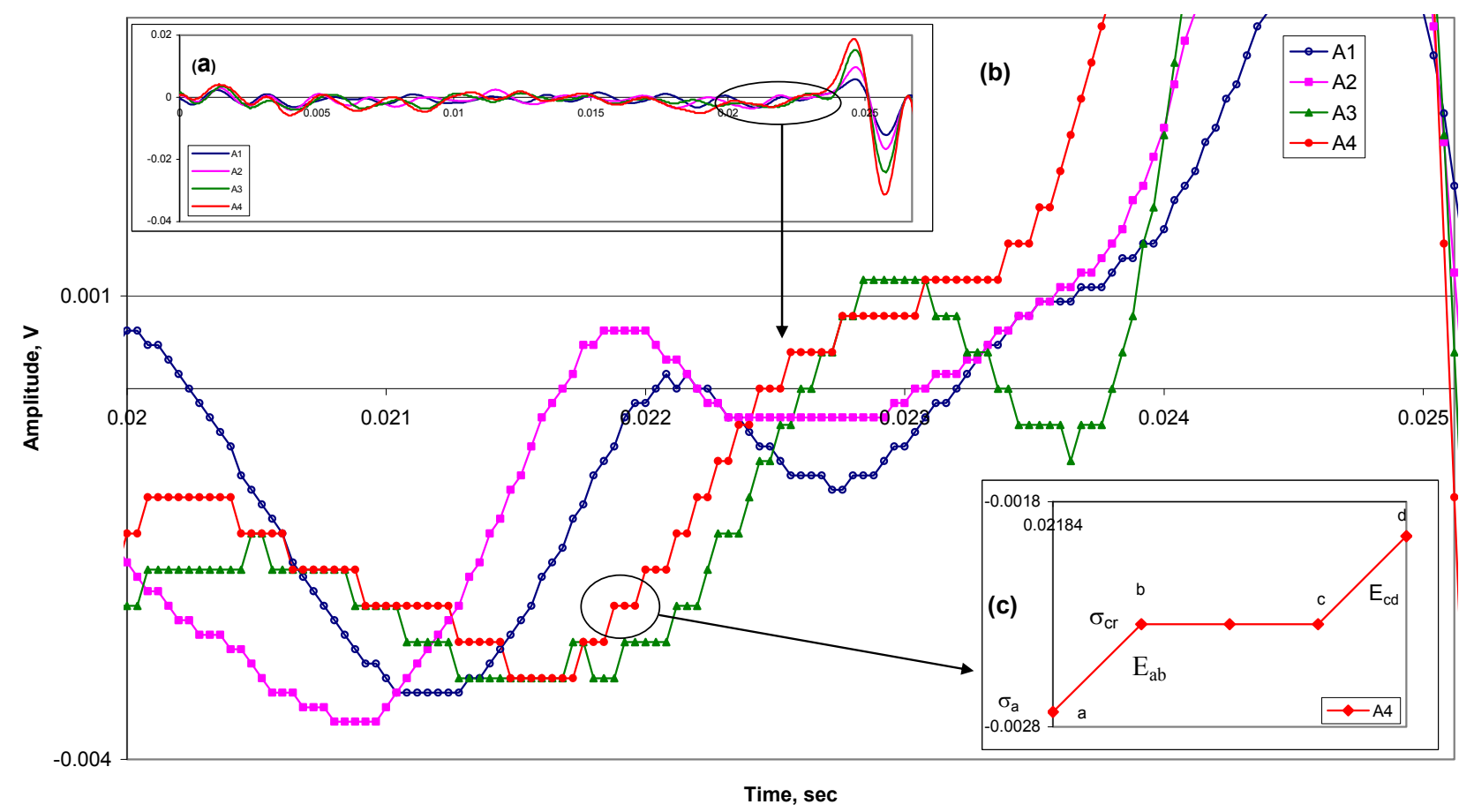

Figure 1. Seismic traces recorded in the $6 \mathrm{~m}-6 \mathrm{~m}$ "source-receiver" location on four amplitude values.

direction: $2-2,6-6$, and 10-10 $\mathrm{m}$. On the short directions, the seismic pulse propagated in the partially saturated rock $(6-6 \mathrm{~m})$ and completely saturated rock $(10-10 \mathrm{~m})$.

The radiator of seismic signals consists of a set of piezoelectric disks. The signal radiates through a liquid spacer and hermetic elastic jacket contacting the borehole wall. Predominant frequency of P-pulse is about $200 \mathrm{~Hz}$. The pressure receiver has the sensor of the piezoelectric type (PDS-21) and, therefore, records compression extension waves. The receiver contacts the borehole wall via an elastic spacer with a liquid. There is a preamplifier with the amplification coefficients $K=100$. The signals were recorded in the digital form high-resolution during of time (Bordo-B-421 system), and were processed on a computer. The digitization time is 8 microseconds and 40 microseconds, the amplitude range is approximately (4$50) \times 10^{-8}$. The discrete amplitude change was fulfiled in a closed cycle, from the minimum to the maximum value and back $\left(\mathrm{A}_{\min } \ldots \rightarrow \mathrm{A}_{\max } \ldots \rightarrow \mathrm{A}_{\min }, 4\right.$ values upward and 3 values downward).

Some parts of seismic trace have the form of ladder with the horizontal steps or plateau. The steps are evidence of interruption in the stress course and the presence of the time delay and deformation delay. The presumable reason of such effect is microplasticity caused by seismic wave. The signs of microplasticity were detected on many seismic records. The typical fragments from seismic traces with seismic microplasticity manifestations (SMM) are presented on Figures $\mathbf{1}$ and 2 .
SMM take place for signals with low and high intensity, i.e. small and great deformation rate.

A SMM example with small strain rate is shown in Figure 1. Here are presented four seismic traces recorded on four amplitude values in the 6-6 m "source-receiver" location. Change in amplitude value during defined time interval determines the amplitude steepness $\mathrm{R}_{\mathrm{A} / \mathrm{t}}$. The amplitude value $A_{i}$ is determined by number $\mathbf{n}$ of the amplitude quantization steps $\left(\mathrm{A}_{\mathrm{qu}}\right): \mathrm{A}_{\mathrm{i}}=\mathbf{n} \times \mathrm{A}_{\mathrm{qu}}$ during one time quantization step, $\mathrm{t}_{\mathrm{qu}}$. The amplitude steepness is calculated as $\mathrm{R}_{\mathrm{A} / \mathrm{t}}=\mathbf{n} \times \mathrm{A}_{\mathrm{qu}} / \mathrm{t}_{\mathrm{qu}}$, i.e. it is determined by way of number $\mathbf{n}$. Thus, steepness is $\mathbf{n}=2$ for amplitudes $A_{1}, A_{2}$ and $\mathbf{n}=5$ for amplitudes $A_{3}, A_{4}$. The step length determines the time delay duration. The time delay in this case is from one $t_{\mathrm{qu}}$ to more than ten of $\mathrm{t}_{\mathrm{qu}}$. On the recordswith the small amplitude steepness, $t_{q u}$ is equal 40 microseconds.

A SMM example of amplitude change with high rate ( $\mathbf{n}=50$ and $\mathbf{n}=100$ ) is presented in Figure 2. This record concerns the case when both source and receiver were located at the depth of $10 \mathrm{~m}$. Here the traces are presented for the upward and downward amplitudes. In principle, there is close coincidence in the form of the repeated traces although there are some nuances (see inset $b$ ). The delay duration in this case is tens of microseconds $\left(\mathrm{t}_{\mathrm{qu}}=8\right.$ microseconds $)$.

The time delay changes the arrival time (see inset $c$ in Figure 2) and can also influence on the wave front duration. In the same time, duration of the time delay depends 


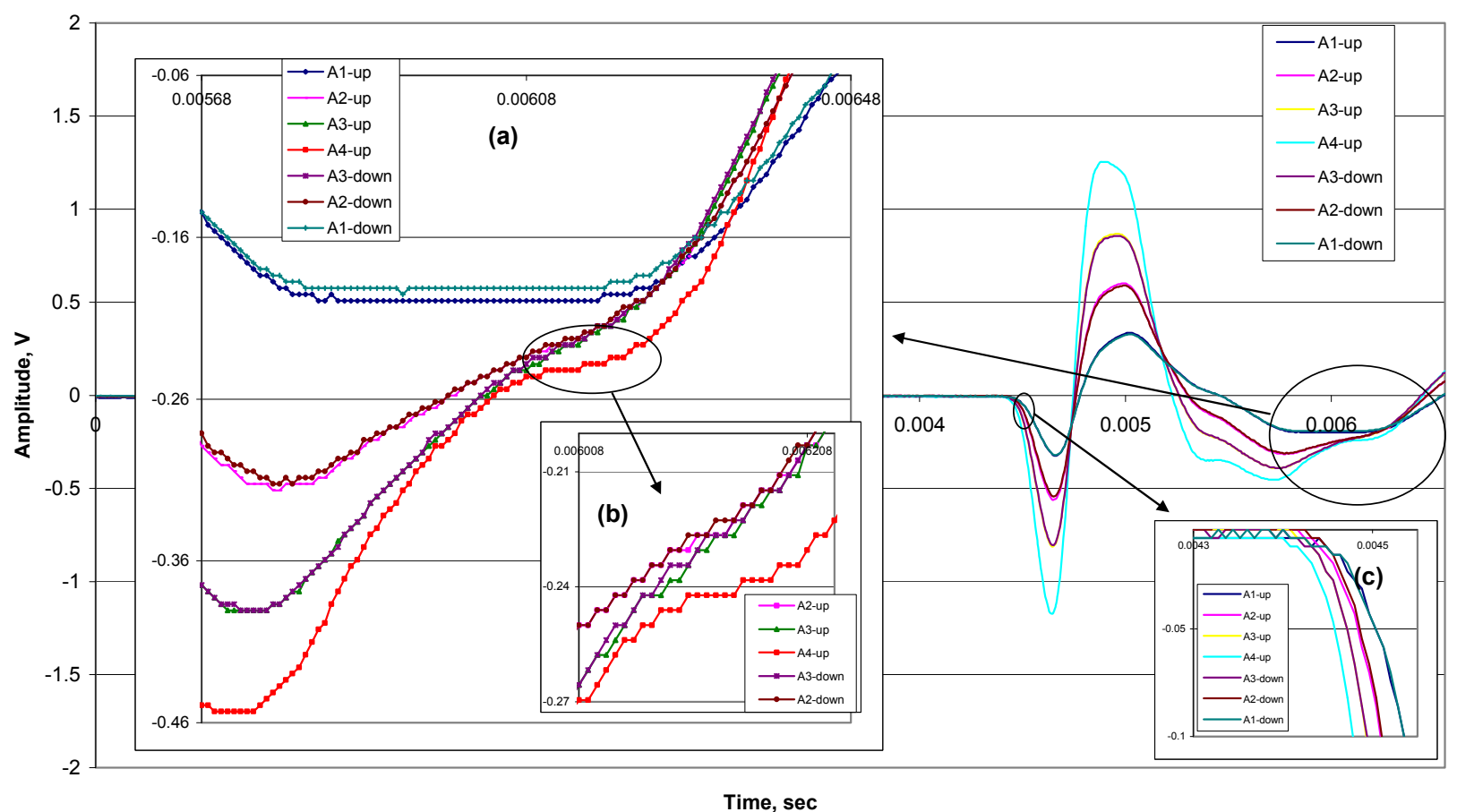

Figure 2. Seismic traces recorded on 4 upward and downward amplitudes in the location of "source- receiver" on $10 \mathrm{~m}$.

on strain amplitude. Therefore, the amplitude variations can lead to the pulse parameters change. The experiments show that the increase in strain amplitude causes the displacement of an arrival time towards smaller time. The protracting of the wave fronts caused by time delay occurs in complex way. In the present time, could say that the intensity increase in wave shows the greater expressiveness of SMM. For the study of these effects, the conducting of the special experiments is needed.

\section{DISCUSSIONS}

Data about dynamic microplasticity in solid states and quasi-static microplasticity in the rocks confirm possibility of SMM conditioned by seismic wave. The strains level in static test and in seismic wave in the moderate amplitude range is approx selfsame $\left(\sim 10^{-6}-10^{-5}\right)$. The difference in the strain rate is not obstacle for the microplasticity process as a physical mechanism in both cases is, seemingly, the same. The point is that microplasticity is the frequency-independent (time-independent) process at least on seismic frequencies. Numerous data testify about prevalence of microplasticity effects in metallic materials, alloys, ceramics, thin-film materials and other solid states in the acoustical and low-frequency range $[18,19]$. Avowed feature of medium with microplasticity is the dependence of wave attenuation on strain amplitude. As regards rock microplasticity, here there are the obvious gaps in one's knowledge. In- asmuch as the direct manifestations of rock microplasticity have been established only during quasi-static stress in lab conditions (measurements on the samples) [10], any study of rock microplasticity under dynamic force is the considerable advancement in these investigations. Especially importantly, when it concerns the dynamic research in natural conditions.

In itself the physical experiment in situ for the purpose of microplasticity detection in the rocks triggered by seismic wave is original. In this experiment, natural medium in which propagation of seismic pulse occurs is test subject. It is unlimited medium in contrast to laboratory samples of small size. This medium consists of the dry and water-saturated rocks. It determines the experiment specificity. Microplasticity detection became possible also thanks to the using of the high-resolution seismic record in the time domain (microseconds) in which there is no need for the usual record of seismic signals. Besides, new information and results originality have been obtained thanks to the sounding by the different-intensity pulses upward and downward. Microplasticity amount depends on the energy level applied. One can surmise that with increasing wave intensity occurs the switching on the sources of microplasticity with the multi-level hierarchy.

SMM can be explained in the following way. Inasmuch as the piezoelectric receiver registers the change in dynamic stress (strain) with time, the flat steps on the trace mean the brief interruption in the course of 
stress. Such stress stop supposes similar stop on the stress-strain curve that can occur owing to the microplasticity process. Total strain $\varepsilon=\varepsilon_{\mathrm{e}}+\varepsilon_{\mu}+\varepsilon_{\mathrm{v}-\mathrm{e}}$ in the small-strain range consists mainly of elastic and microplastic component $\left(\varepsilon_{\mathrm{e}}+\varepsilon_{\mu}\right)$, as viscoelastic component $\varepsilon_{\mathrm{v}-\mathrm{e}}$ is comparatively small [10,11]. During loading (or unloading), both components have own contribution to the total strain. The stop in the course of stress occurs when the stress reaches some critical value $\sigma_{\mathrm{cr}}$ (for example, in the point b see inset (b) in Figure 1). At that moment, the redistribution of contribution between components occurs thus that contribution of the microplastic component become predominant. In the extreme case, the strain increment occurs only thanks to microplasticity as the increment due to the elastic deformation does not occur at all [10]. Therefore, the yield process is also possible. The regrouping in components occurs thus that effective modulus $\mathrm{E}_{\mathrm{ab}}$ and accordingly the stress and strain remain invariable during interval $\mathbf{b}-\mathbf{c}$. It is possible owing to the distinctive feature of rock microplasticity that can both increase and decrease or even vanish during stress. In the point c after delay, the stress recommences own course with the same modulus. There is the hierarchical set of the diverse critical stresses that switch on the microplasticity sources when amplitude increases (decreases). Thus, seismic wave switches on the process of the anomalous redistribution of stresses and strains and respectively adequate to it process of the structural realignment in the rock. It takes a definite time that leads to deformation delay. In order to exclude the instrumental factor in appraisal of SMM, the amplitude error of analog-digital converter is checked. Testing of this device shows that the own error in plateau duration does not exceed 2 microseconds in the broad range of the amplitude steepness.

Similar deformation stops caused by microplasticity were detected in $\mathrm{C}_{60}$ single crystals [20]. It is shown that magnetic field manipulations lead to a change in the strain rate, the decrease in the rate being accompanied by a brief interruption of deformation. The deformation delay (incubation period) was observed also in the high-temperature superconductors, possessing by microplasticity [21]. Delay appearance is bound with presence of opposite internal stresses for decrease of which time is required. The wave attenuation mechanism in the rock with microplasticity (referring to the experiments [10]) is theoretically substantiated also in work [22]. As regards the mechanism of wave attenuation, the combination of known mechanisms is possible, for instance, as the hybrid relaxation-hysteresis mechanism [12]. It must not be ruled out also the acoustoplastic effect in the rocks as in metals and alloys [23]. The instance of stress jumps marked on seismic record evokes the great caution in interpretation of this effect, and therefore for the time being, we do not examine this question.

\section{CONCLUSIONS}

The result of this work is the new knowledge about nature of propagation of the mechanical oscillations in the Earth. The unnoticed heretofore presence in seismic record of microplasticity manifestations was detected thanks to the high-resolution signals measurements. The quasi-static microplasticity of the rocks, dynamic microplasticity of many solid states denoted the prospect of our search. The dynamic microplasticity in the rocks definitely is bound with critical amplitudes but its dependence on strain rate is not yet established. One may surmise data about rock microplasticity will enlarge the comprehension of some known effects that had not formerly of the satisfactory physical explanation. We see it now in the delay effect of the arrival time and the pulse shortening-widening.

As stated above, the nature of microplasticity in the rocks can coincide with known mechanisms in the solid states (for instance, dislocation microplasticity) or to be quite other (quasi-microplasticity). In the last case in spite of the difference in mechanism, the microplasticity manifestations can be the same as in usual solid states. This question requires subsequent clarification. Microplasticity affects the little-known anelastic processes of the small-amplitude wave propagation. The new knowledge about nonlinear-anelastic processes during wave propagation will help in discovery of new diagnostic indications permissive to increase the efficiency of seismic method for search of oil-gas deposits.

\section{ACKNOWLEDGMENT}

The author thanks G.V. Egorov for the help in the experimental work.

\section{REFERENCES}

[1] Mavko, G.M. (1979) Friction attenuation: An inherent amplitude dependence. Journal of Geophysical Research, 84(9), 4769-4775.

[2] Winkler, K.W., Nur, A. and Gladwin, M. (1998) Friction and seismic attenuation in rock. Nature, 274, 528-531, 1979.

[3] Tutuncu, A.N., Podio, A.L. and M.M. (1994) Sharma An experimental investigation of factors influencing compressional- and shear-wave velocities and attenuations in tight gas sandstones. Geophysics, 59(1), 77-86.

[4] Ostrovsky, L.A. and Johnson, P.A. (2001) Dynamic nonlinear elasticity in geomaterials. La Rivista del Nuovo Cimento, 24(4), 7.

[5] Johnston, D.H. and Toksoz, M.N. (1980) Thermal cracking and amplitude dependent attenuation. Journal of 
Geophysical Research, 85, 937-942.

[6] Mashinskii, E.I., Koksharov, V.Z. and Nefedkin, Y.A. (1999) Amplitude-dependent effects in the range of small seismic strains. Geologiya i Geofizika, 40, 611-618.

[7] Mashinskii, E.I. (2004) The variants of the strainamplitude dependence of elastic wave velocities in the rocks under pressure. Journal of Geophysics and Engineering, 1, 295-306.

[8] Mashinskii, E.I. (2007a) Effect of strain amplitude on the relaxation spectra of attenuation in the dry and saturated sandstone under pressure. Journal of Geophysics and Engineering, 4, 194-203.

[9] Zaitsev, V.Y., Nazarov, V.E. and Talanov, V.I. (1999) Experimental study of the self-action of seismoacoustic waves. Acoustic Physics, 45(6), 720-726.

[10] Mashinsky, E.I. (1994) Quasi-micro-plasticity processes and nonlinear seismicity. Physics of the Solid Earth, 30, 97-102.

[11] Mashinskii, E.I. (2005b) Non-linear stress-strain relation in sedimentary rocks and its effect on seismic wave velocity. Geophysica, 41(1,2), 3-17.

[12] Mashinskii, E.I. (2006) Nonlinear amplitude-frequency characteristics of attenuation in rock under pressure. Journal of Geophysics and Engineering, 3, 291-306.

[13] Mashinskii, E.I. (2005a) Experimental study of the amplitude effect on wave velocity and attenuation in consolidated rocks under confining pressure. Journal of Geophysics and Engineering, 2, 199-212.

[14] Mashinskii, E.I. (2008) Amplitude-frequency dependencies of wave attenuation in single-crystal quartz: Experimental study. Journal of Geophysical Research, 113, B11304.

[15] Xu, H., Day, S.M. and Minster, J.-B.H. Model for nonlinear wave propagation derived from rock hysteresis measurements. Journal of Geophysical Research, 103(B12),
29,915-29,929.

[16] Braccini, S., et al. (2000) The maraging-steel blades of the Virgo super attenuator. Measurement Science and Technology, 11, 467-476.

[17] Mashinskii, E.I. (2007) Amplitude-dependent effects of Longitudinal Seismic Wave Propagation in the Interhole Space. Izvestya, Physics of the Solid Earth, 43(8), 683-690.

[18] Nishino, Y. and Asano, S. (1996) Amplitude-dependent internal friction and microplasticity in thin-film materials. Journal de Physique IV, 6, 783-786.

[19] Golovin, I.S., Sinning, H.-R., Goken, J. and Riehemann, W. (2004) Fatigue-related damping in some cellular metallic materials. Materials Science and Engineering, A370, 537-541.

[20] Smirnov, B.I., Shpeizman, V.V., Peschanskaya, N.N. and Nikolaev, R.K. (2002) Effect of magnetic field on microplastic strain rate for $\mathrm{C}_{60}$ single crystals. Physics of the Solid State, 44(10), 2009-2012.

[21] Davies, P.W. and Wilshire, B. (1971) On internal stress measurement and the mechanism of high temperature creep. Scripta Metallurgica, 5(6), 475-478.

[22] Yarushina, V.M. and Podladchikov, Y.Y. (2008) Micriscale yielding as mechanism for low-frequency imtrinsic seismic wave attenuation. $70^{\text {th }}$ EAGE Conference \& Exhibition, Rome, Italy, 9-12 June.

[23] Sapozhnikov, K.V., Vetrov, V.V., Pulnev, S.A. and Kustov, S.B. (1996) Acousto-pseudoelastic effect and internal friction during stress-induced martensitic transformations in $\mathrm{Cu}-\mathrm{Al}-\mathrm{Ni}$ single crystals. Scripta Materialia, 34(10), 1543. 